\title{
Impact of Deposit Recoat Cycle Length on Hot Corrosion of CMSX-4
}

\author{
Joy Sumner ${ }^{1} \cdot$ Quentin Aksoul $^{1} \cdot$ Joemar Delgado $^{1}$ • \\ Andrew Potter $^{1} \cdot$ Simon Gray ${ }^{1}$
}

\begin{abstract}
Hot corrosion causes significant problems for both aerospace and power generation industries, where the combination of high temperature, corrosive gases, and contaminants severely limits component operating lifetimes in gas turbine hot gas streams. Multiple laboratory testing methodologies exist to study this hot corrosion, and these can be affected by a range of variables. This paper investigated the impact of varying deposit recoat cycle length when using the 'deposit recoat' testing method. CMSX-4 samples were exposed to simulated type II (pitting) hot corrosion conditions, with the same overall deposit load (averaged across the total exposure run), but different deposit recoat cycles. Post-exposure, samples underwent dimensional metrology analysis to compare metal loss resulting from different deposit recoat cycle lengths. Results for CMSX-4 suggest very small differences in corrosion losses, indicating CMSX-4 hot corrosion datasets obtained from deposit recoat experiments with different deposit recoat cycle lengths can be compared with confidence.
\end{abstract}

Keywords Deposit recoat period - Type II hot corrosion - CMSX-4 - Dimensional metrology

Joy Sumner

j.sumner@cranfield.ac.uk

Andrew Potter

a.potter@cranfield.ac.uk

Simon Gray

s.gray@cranfield.ac.uk

1 Cranfield University, Cranfield, Bedfordshire MK43 OAL, UK 


\section{Introduction}

Hot corrosion is a problem for components (e.g., blades, vanes, combustor cans) exposed to the hot gas path of both aerospace and power generation gas turbines. Both air and fuel contain a range of potential contaminants (sulfur, alkali metals, etc) which react in the high temperature, combusted gas stream. In such environments, corrosive deposits can condense out of the gas stream and onto the relatively cool components, where, coupled with $\mathrm{SO}_{2} / \mathrm{SO}_{3}$ gaseous species, fluxing of protective oxide scales can occur (hot corrosion incubation) followed by base alloy attack (hot corrosion propagation) [1]. Typically, this degradation mechanism is characterized as either type I (broad front and internal sulfidation, and taking place at temperatures $\sim 900{ }^{\circ} \mathrm{C}$ ) or type II (pitting attack, often about $700{ }^{\circ} \mathrm{C}$ ) [2].

Due to the industrial importance of this degradation mechanism, a range of different testing methodologies have been developed and have been integrated into ISO standards [3-5]. Common hot corrosion simulation methods can be classified as engine testing; pilot-scale testing; or laboratory-scale testing. In the first two classes, samples are cold relative to the gas environment they are immersed in, and as such there is a driving force for the deposition of the required corrosive species from this environment [6]. By contrast, in many laboratory tests, there is no heat flux between the samples and environment, and so deposits must be formed in different manners. Techniques such as Dean's Rig rely on supersaturation of the deposit relative to the gas steam [7], while other systems periodically recoat the deposit on the sample ('deposit recoat' technique) [8].

In simulated testing, the hot corrosion mechanism is sensitive to a large number of variables, including deposit chemistry, deposition flux, concentration of corrosive gas, and varying temperature range $[9,10]$. Thus, it is important to understand the effect of these when attempting to simulate representative environments and corrosion behavior in laboratory tests.

This paper studies the impact of varying deposit recoat cycle length in 'deposit recoat' test set-ups; a variable which is not often reported. In particular, it investigates whether varying the length of deposit recoat cycles, while retaining the same overall deposition flux, greatly affects the hot corrosion damage rate and thus the good metal loss for CMSX-4 superalloy samples.

\section{Experimental Procedures}

CMSX-4 (composition in Table 1) was machined into long bars of connected 'cotton reels' of approximate diameter $10 \mathrm{~mm}$ and 'cotton reel' length $10 \mathrm{~mm}$ (Fig. 1). Individual 'cotton reels' were separated off, and their exact diameters,

Table 1 Nominal composition of CMSX-4 (wt \%)

\begin{tabular}{lllllllllll}
\hline Alloy & $\mathrm{Ni}$ & $\mathrm{Co}$ & $\mathrm{Cr}$ & $\mathrm{Ti}$ & $\mathrm{W}$ & $\mathrm{Al}$ & $\mathrm{Re}$ & $\mathrm{Ti}$ & $\mathrm{Mo}$ & $\mathrm{Hf}$ \\
\hline CMSX-4 & Bal. & 9.0 & 6.5 & 6.5 & 6.0 & 5.6 & 3.0 & 1.0 & 0.6 & 0.1 \\
\hline
\end{tabular}




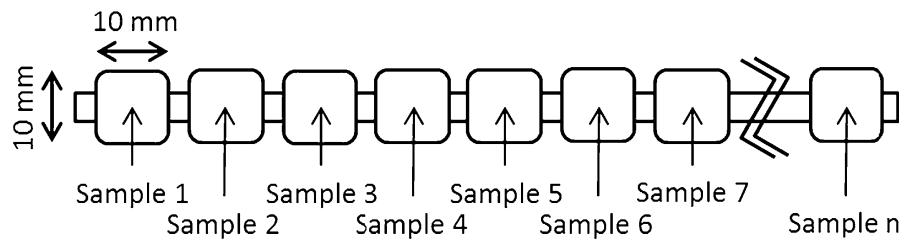

Fig. 1 Several 'cotton reel' samples were produced from one rod, then cut off prior to use

heights, and weights recorded before testing as machined $\left(R_{\mathrm{a}}<0.4 \mu \mathrm{m}\right)$. For these pre-exposure measurements, the micrometer had a resolution of $\pm 1 \mu \mathrm{m}$ and the analytical balance had a resolution of $\pm 0.01 \mathrm{mg}$.

Type II hot corrosion conditions were simulated using the 'deposit recoat' technique [11]. Up to 24 samples at a time can be placed into a vertical, controlled atmosphere furnace (hot zone controlled to $\pm 5{ }^{\circ} \mathrm{C}$ using an R-type thermocouple). This furnace contained alumina furniture to prevent reaction with the corrosive environment generated and potentially act as a catalyst for the formation of $\mathrm{SO}_{3}$. The test was conducted at $700{ }^{\circ} \mathrm{C}$ in a gas environment of $300 \mathrm{vpm} \mathrm{SO}_{\mathrm{X}}$ in air (flow rate of $50 \mathrm{~cm}^{3} / \mathrm{min}$ ). A total length for each test was set at $300 \mathrm{~h}$ (sufficient time for the CMSX-4 to have entered propagation [12]).

Deposits of $2 \% \mathrm{NaCl}$ in $\mathrm{Na}_{2} \mathrm{SO}_{4}$ were applied to the sample with a flux of $5 \mu \mathrm{g} /$ $\mathrm{cm}^{2} / \mathrm{h}$. This flux was adjusted for the exact sample size (as determined using the micrometer measurements) and the deposit recoat cycle length (either 25, 100, or $300 \mathrm{~h}$ not including the heat-up/cool-down time). The variable deposit recoat cycles are shown in Fig. 2. Deposit recoating was conducted at room temperature (at the same time as weight change was recorded). For a total test of $300 \mathrm{~h}, 1.5 \mathrm{mg} / \mathrm{cm}^{2}$ of deposit would have been applied in up to 12 different steps (for 25 -h deposit recoat cycle lengths). Thus, for 25 -h deposit recoat cycle lengths, the deposit was reapplied 12 times, with a dose of ' 1 ' $\left(125 \mu \mathrm{g} / \mathrm{cm}^{2}\right)$; for 100 -h deposit recoat cycle lengths, the deposit was reapplied three times, with a dose of ' 4 ' $\left(500 \mu \mathrm{g} / \mathrm{cm}^{2}\right)$; and for the $300-\mathrm{h}$ deposit recoat cycle, the deposit was applied one time, with a dose of ' 12 ' $\left(1.5 \mathrm{mg} / \mathrm{cm}^{2}\right)$.

To enable consideration of any unintended thermal cycling effects (and related stresses), additional specimens were tested with the same range of cycle lengths, but with all the deposit applied at the start of the 300-h test exposure (equivalent to a dose of ' 12 ' or $1.5 \mathrm{mg} / \mathrm{cm}^{2}$ ).

Repeat samples were run in the tests, such that two samples could be removed for post-exposure analysis at 100-, 200-, and 300-h exposure periods. This enabled a comparison of sample-to-sample variation in the test runs.

Post-exposure, metal loss/good metal loss data were collected from the sample's cross section (retaining any surface scales/deposit) using a dimensional metrology technique $[3,12,13]$. In this technique, pre- and post-exposure sample dimensions are compared (with an accuracy of $\pm 5 \mu \mathrm{m}$ ) using an optical microscopy-based technique to plot the metal loss (ML) or good metal loss (GML) from a statistically significant number of locations around each individual sample (here 30 locations). GML is defined as the ML plus any internal damage, where present. In instances 


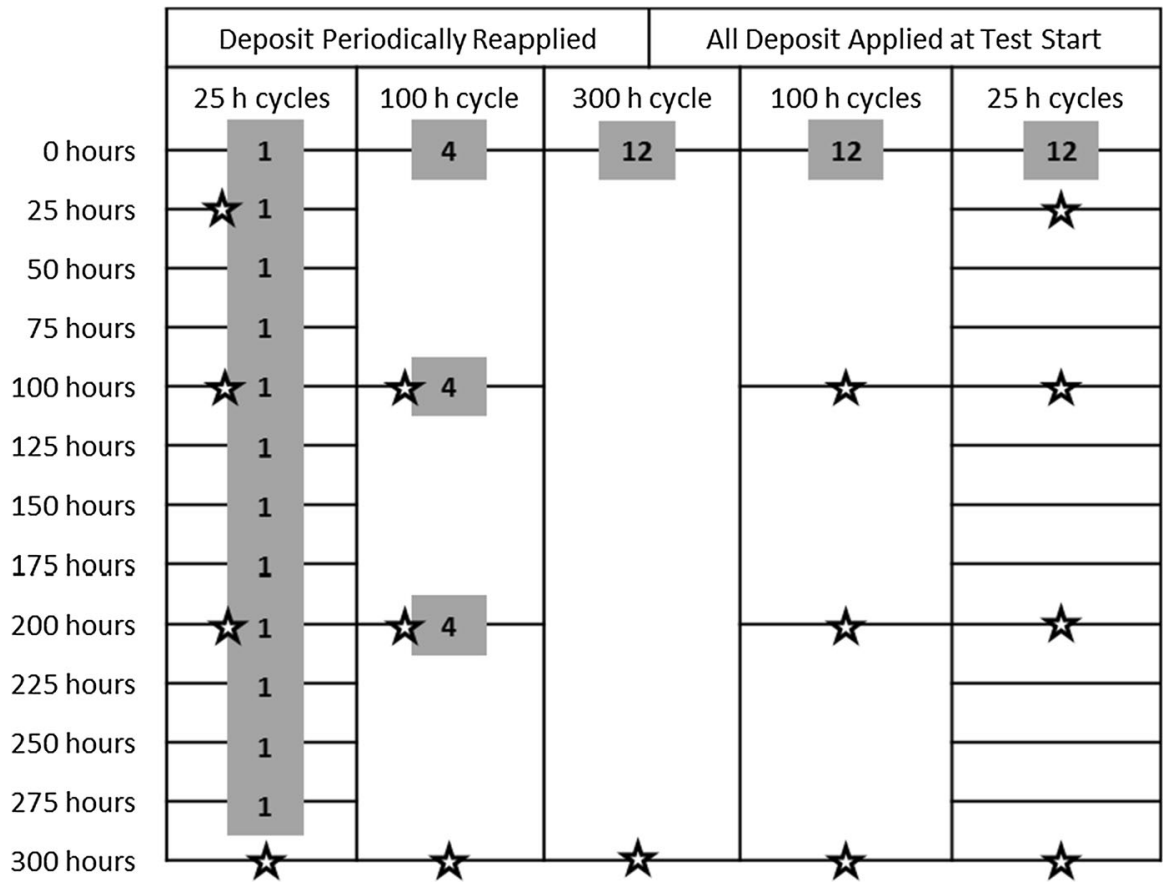

Fig. 2 Schematic of the test matrix. Stars indicate removal of two samples for destructive investigation (except for the 25-h removal, where just one sample was removed; and the 300-h cycle, where four samples were removed). Numbers in gray indicate 'dose' of deposit applied at specific times (summing to a total dose of ' 12 ' over the full 300-h test)

where no internal damage is observed, GML and ML will give identical measurements.

Following data collection, the dataset is reported from greatest to smallest extent of GML and plotted against cumulative probability. Cumulative data can be plotted for a range of probability distributions. One of the more common is as a percentage of the data recorded; however, here the data here have been plotted against cumulative normal probability with units of standard deviation from the median (std. dev.). These have been calculated using Excel functions for standard normal cumulative probability. One advantage of plotting the GML against normal cumulative probability is that any underlying normal distribution(s) in the GML data will appear to be plotted as straight lines, and different normally distributed damage mechanisms may appear as straight lines of different gradient. Once produced, these datasets allow easy comparison between different samples (e.g., to compare the impact of different deposit recoat cycle lengths).

In addition to the dimensional metrology GML data, weight change data were collected. Corrections were made for increases in applied deposit weight when recoating. 


\section{Results}

\section{Initial Results from Different Deposit Recoat Cycle Lengths}

Optical micrographs (examples in Fig. 3) show that, as expected for type II hot corrosion, no internal damage is observed. Samples show smooth metal interfaces, either covered with deposit/oxide scales or without (where such scales have spalled). Individual pits are not often observed. This has previously been noted $[12,14]$ and is thought to result from rapid metal loss causing pits to connect up, producing attack with an apparent broad front. That significant metal loss occurred can be inferred from the outer metal interface which, at this magnification, has a 'wavy' rather than smooth (as machined) appearance.

Mass change data (Fig. 4) show that, for samples with regular reapplication of deposit, generally a decrease in sample weight can be observed. This is consistent with oxide spallation from the sample surface. For samples with the entire deposit dose applied at the start of the test, initial increases in weight (having allowed for the weight of the deposit) may be measured before weight loss due to spallation occurs. For all samples, non-zero weight changes are recorded within the first $25 \mathrm{~h}$, indicating no/negligible incubation period. This is consistent with earlier work $[12,14]$.

In general, there is little variation between the final, $300 \mathrm{~h}$, weight change for samples with different cycle lengths, although samples which have received the full deposit dose at the test start appear to show greater scatter in weight change data.

It should be noted that weight change data are often an unreliable metric for hot corrosion damage, where the balance of loss (due to processes such as spallation or
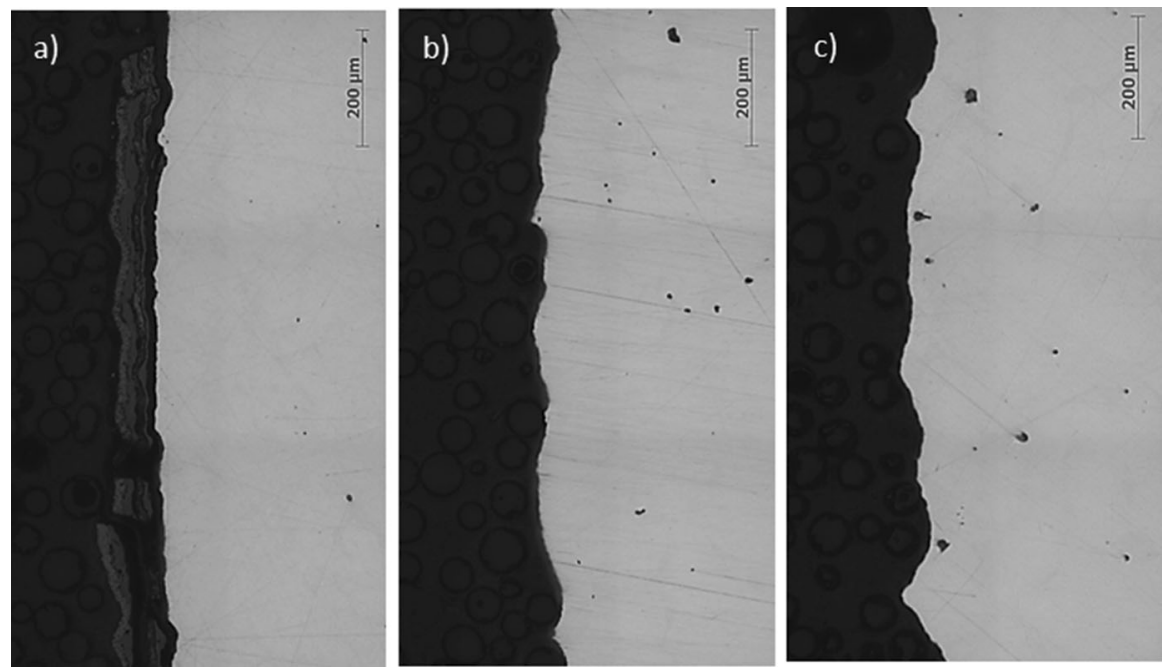

Fig. 3 Optical micrographs of the CMSX-4 samples exposed for $300 \mathrm{~h}$ at $700{ }^{\circ} \mathrm{C}$, with periodic deposit recoats. Deposit recoat cycle lengths: a $25 \mathrm{~h}, \mathbf{b} 100 \mathrm{~h}$, and c $300 \mathrm{~h}$ 

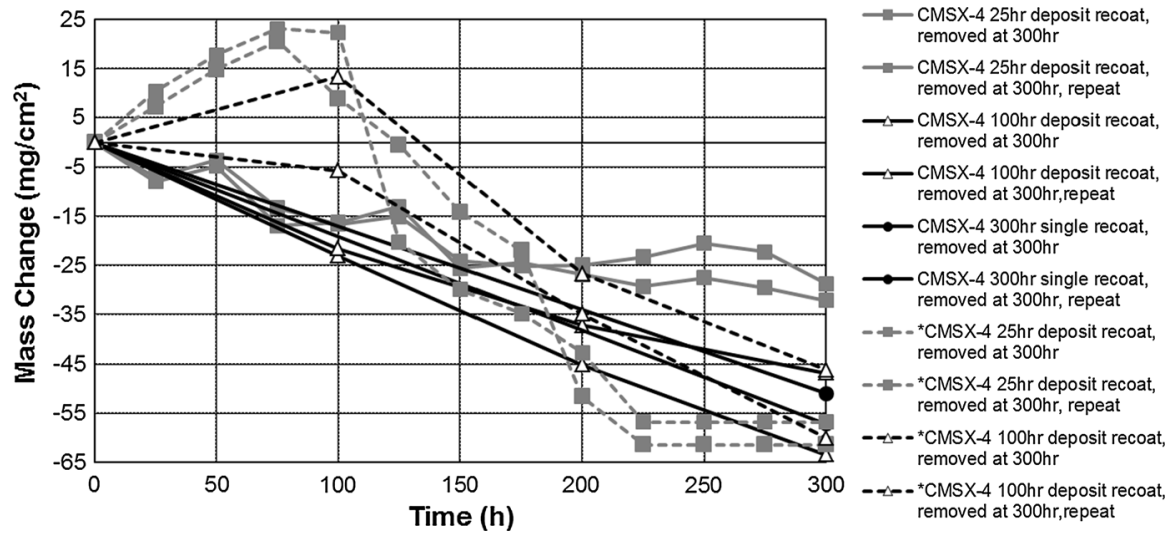

Fig. 4 Mass change data from samples exposed for $300 \mathrm{~h}$, with different deposit recoat cycle lengths. Samples with regular deposit recoats have solid lines; samples with all deposit applied at exposure start have dashed lines (and are marked with asterisk in the legend)

evaporation) and gain (from processes such as oxidation and corrosion) can be difficult to interpret [14].

Figure 5 gives an example of the dimensional metrology data collected from samples exposed with 25 -h deposit recoat cycles. Where the deposit has been periodically reapplied, the median GML value increased from $\sim 25 \mu \mathrm{m}$ after $100-\mathrm{h}$ exposure to $\sim 58 \mu \mathrm{m}$ after 200 -h and $\sim 87 \mu \mathrm{m}$ after 300 -h exposure. The spread in GML around the sample remained constant at $\sim 40 \mu \mathrm{m}$. The CMSX-4 samples moved rapidly into propagation; by $100 \mathrm{~h}$ all measured locations on both (repeat) 100-h samples show non-zero GML values, indicating that they had ceased to incubate and that the base alloy was under attack.

By contrast, when all of the deposit was applied at the start of the test, larger initial median GML values were recorded ( $\sim 40 \mu \mathrm{m}$ after $100-\mathrm{h}$ exposure). This increased to $\sim 72 \mu \mathrm{m}$ after $200 \mathrm{~h}$. However, in the final series of deposit recoat cycles to $300-\mathrm{h}$ total exposure, the GML only increased to $\sim 77 \mu \mathrm{m}$.

Similar GML data are given in Fig. 6 for samples with 100-h deposit recoat cycles. However, for these longer cycles, both tests with periodic recoat of deposit and tests with all deposit initially applied show similar trends. There was an initial rapid loss in GML (after $100 \mathrm{~h}, \sim 37 \mu \mathrm{m}$ median GML for samples with periodic deposit recoat and $\sim 30 \mu \mathrm{m}$ for samples with all deposit initially applied). Further GML is recorded after 200-h exposure (to $\sim 78 \mu \mathrm{m}$ median GML for samples with periodic deposit recoat and $\sim 67 \mu \mathrm{m}$ for samples with all deposit initially applied). However, until the end of the test, there was little further GML for samples with periodic deposit recoat ( $\sim 9 \mu \mathrm{m}$ increase, up to $\sim 87 \mu \mathrm{m}$ median GML) or for samples with all deposit initially applied ( $\sim 6 \mu \mathrm{m}$, to $\sim 73 \mu \mathrm{m}$ median GML).

Finally, Fig. 7 shows the impact of applying all of the deposit at the start of the test, and running without cycling. In total, four samples have run, uninterrupted, for $300 \mathrm{~h}$ with an effective deposit dose of '12.' Two samples were in the same furnace used previously for the tests with variable deposit recoat cycle length, and two 


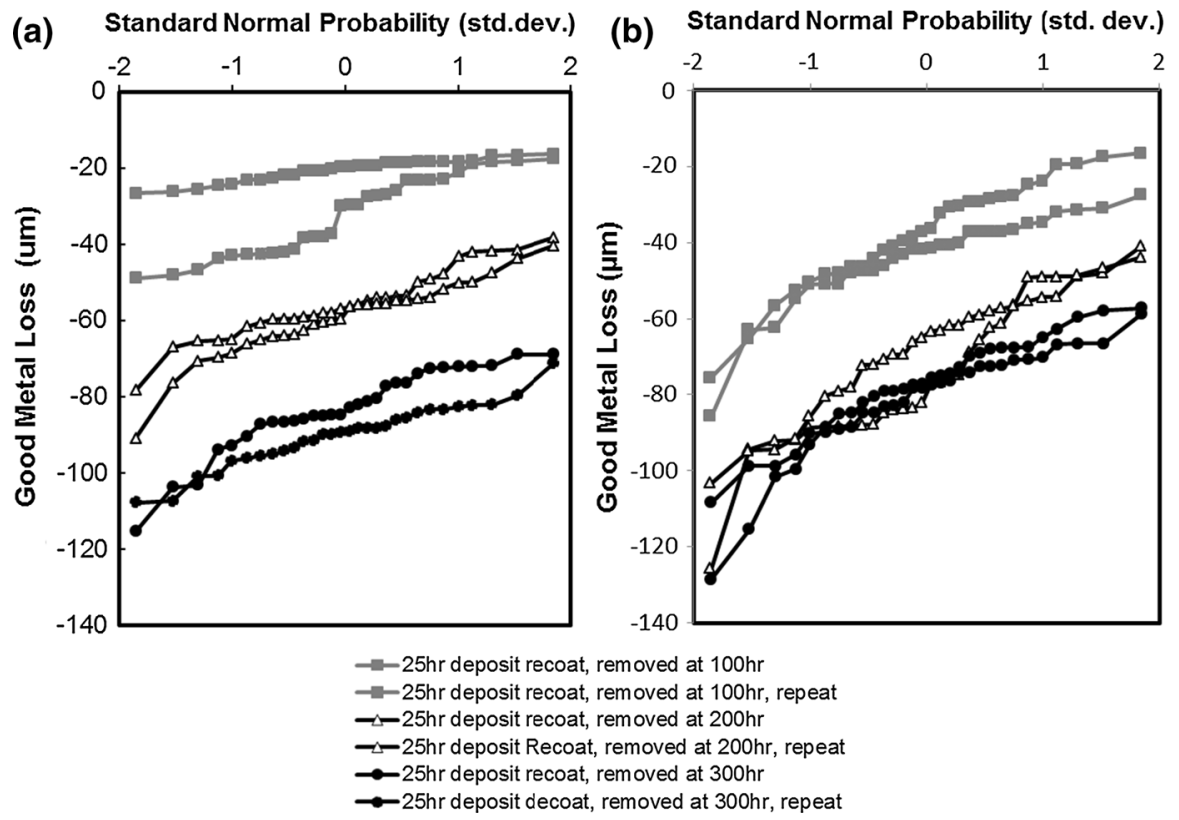

Fig. 5 GML dimensional metrology data: 25-h deposit recoat cycles. a Samples with regular deposit recoats and b samples with all deposit applied at exposure start. Gray square symbols from samples removed after $100 \mathrm{~h}$; white triangle symbols from samples removed after $200 \mathrm{~h}$; and black circle symbols from samples removed after $300 \mathrm{~h}$

samples were exposed in the same furnace used previously for the tests where all deposit was initially applied and only the thermal cycle changed. Median GML values of $\sim 100 \mu \mathrm{m}$ are recorded. Larger variation between the median GML values of the two 'repeat' test samples is seen in one of the tests $(26 \%)$. However, the spread in GML for all four 300-h cycle samples covers a similar range.

It is not thought that the variation in GML for the 300-h cycle samples is due to differences in the two furnaces used, as three of the four samples have very similar GML values. Additionally, the furnaces had an identical setup and had undergone thermal profiling to ensure that samples experienced identical temperatures. The variation of the fourth sample to the others does, however, emphasize the need to use multiple samples.

The GML data for the different samples are summarized in Fig. 8, which plots the increase in median GML value with increasing exposure time. These median values show little variation between the different deposit recoat cycle lengths. Where all of the sample's deposit was applied at the start of a test, there is a slight increase in scatter of data after $200 \mathrm{~h}$, but this falls again for samples destructively analyzed after $300 \mathrm{~h}$. It should be noted that only median GML values are given, not spreads in metal loss. 
(a) Standard Normal Probability (std.dev.)

(b) Standard Normal Probability (std. dev.)
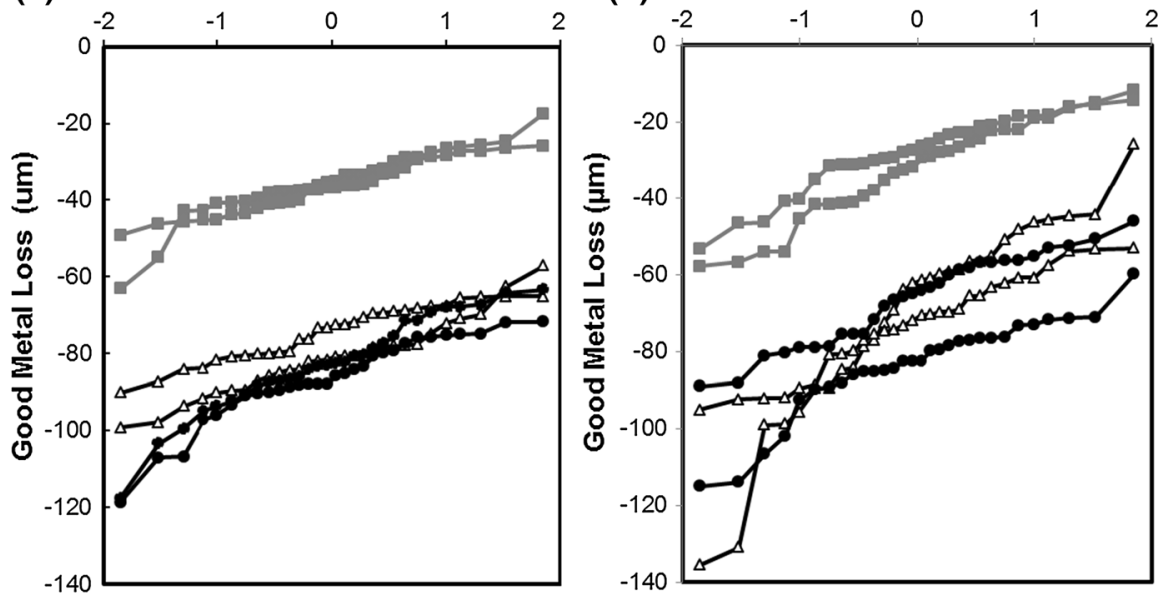

$-100 \mathrm{hr}$ deposit recoat, removed at $100 \mathrm{hr}$

$-100 \mathrm{hr}$ deposit recoat, removed at $100 \mathrm{hr}$, repeat

$\square-100 \mathrm{hr}$ deposit recoat, removed at $200 \mathrm{hr}$

$\checkmark 100 \mathrm{hr}$ deposit recoat, removed at $200 \mathrm{hr}$, repeat

$\rightarrow 100 \mathrm{hr}$ deposit recoat, removed at $300 \mathrm{hr}$

$\multimap 100 \mathrm{hr}$ deposit recoat, removed at $300 \mathrm{hr}$, repeat

Fig. 6 GML dimensional metrology data: 100-h deposit recoat cycles. a samples with regular deposit recoats and b samples with all deposit applied at exposure start. Gray square symbols from samples removed after $100 \mathrm{~h}$; white triangle symbols from samples removed after $200 \mathrm{~h}$; and black circle symbols from samples removed after $300 \mathrm{~h}$

(a) Standard Normal Probability (std.dev.)

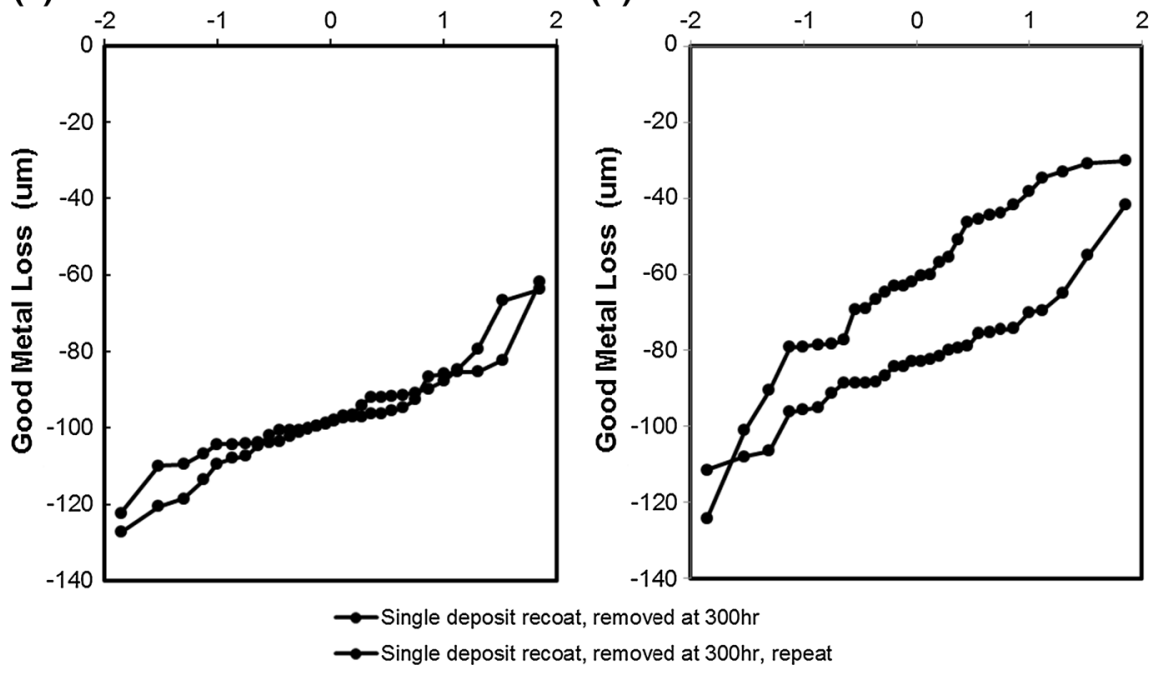

Fig. 7 GML dimensional metrology data: 300-h deposit recoat cycles. a Samples in furnace previously used for deposit recoat cycle variation and $\mathbf{b}$ samples in furnace previously used only for thermal cycle variation 


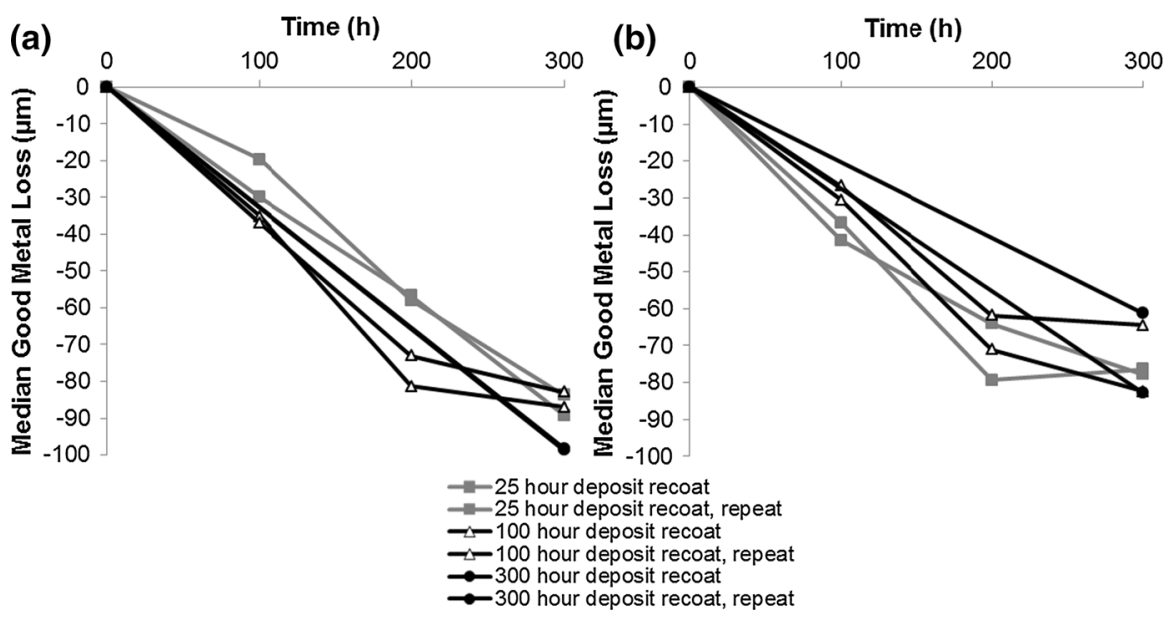

Fig. 8 Trend in median GML over time from the dimensional metrology data for samples with various deposit recoat cycle lengths. a Samples with regular deposit recoats and b samples with all deposit applied at exposure start

\section{Discussion}

\section{The Effect of Changing Deposit Recoat Cycle Length}

Initially, it was expected that changes in corrosion rate would occur with changes in deposit recoat cycle length. The nature of these changes could have resulted in either slower corrosion, due to deposit evaporation/exhaustion, or faster corrosion due to more rapid initial attack under the higher deposit dose. For CMSX-4, this was not clearly observed; this could be due to a balance of these two factors.

Where the deposit was regularly replenished (e.g., every $25 \mathrm{~h}$ ), the GML rate appeared fairly constant at $\sim 25$ to $29 \mu \mathrm{m}$ every $100 \mathrm{~h}$. However, as the deposit recoat cycle length increased (to $100 \mathrm{~h}$ ), the rate of GML became more difficult to define. This may be due in part because, by $100 \mathrm{~h}$, the samples were undergoing significant metal attack and the corrosive deposit might have been lost via spallation.

Of the three different cycle lengths tested with regular deposit reapplication (25, 100, and $300 \mathrm{~h}$ ), all three appeared to result in similar GMLs after the full 300-h exposure period. Furthermore, all samples showed similar spreads in measured GML (from $\sim 60$ to $\sim 120 \mu \mathrm{m}$ ) across the measurements taken.

Where the entire deposit load was applied initially (rather than with periodic recoats), more rapid GML accumulation over short exposure times did occur. This is consistent on work looking at the impact of changing deposit fluxes; as the flux increases, so too does the rate of hot corrosion [12]. These samples effectively experienced very high initial fluxes. However, as the total exposure length increased, and the deposit was not reapplied, the total median GML experienced by samples where the entire deposit load was initially applied appeared to slow relative to samples with regular deposit application (e.g., Fig. 8). This potentially implies 
some change in the corrosion environment aggressiveness as discussed in the next section. Possibly if the test had run for longer, the impact of not reapplying the deposit would have become obvious.

It is worth noting that, in Fig. 8, after $\sim 300 \mathrm{~h}$, all samples appeared to show similar median GML values. More variation in extreme value GML in certain conditions was observed. It could be coincidence that the more rapid initial GML rates of the samples with the initial application of the entire deposit load then slowed sufficiently that the periodically recoated samples 'caught up.' Another possible explanation is that factors such as the increasing thickness of the scale slowed additional reactions.

\section{Routes for Deposit Exhaustion}

The variability/slowing in GML with increasing cycle length potentially implies changes to the conditions necessary for hot corrosion to take place, in this case deposit exhaustion. As mentioned in the previous section, several possible routes exist for the loss of the necessary corrosive deposit:

1. Evaporation of the deposit

2. Spallation of the deposit along with other associated scales

3. Chemical exhaustion of the deposit

\section{Evaporation of the Deposit}

For a deposit to exist upon a sample surface, there must be a driving force for it to remain. In most instances, this is affected by the temperature differential between the sample and the gas, which in turn affects the partial pressure of the species in the deposit and saturation partial pressure(s) [6]. In furnace testing, where sample and gas are at comparable temperatures, there is the potential for evaporation of any applied deposit, especially in flowing gases, where saturation pressures are unlikely to be reached.

\section{Spallation of the Deposit Along with Other Associated Scales}

Images of the samples (Fig. 9) show that the CMSX-4 samples were undergoing sever hot corrosion attack early on. Large portions of the sample surface have spalled and, as the deposit will have been applied to this surface, is it possible that large fractions of the deposit were lost at this time, effectively lowering the deposit flux and thus the hot corrosion attack rate.

\section{Chemical Exhaustion of the Deposit}

Where reaction products are not frequently replaced, there is the possibility of depletion and thus slowing reaction rates.

It is likely that all of these processes are taking place in parallel. 
(a)

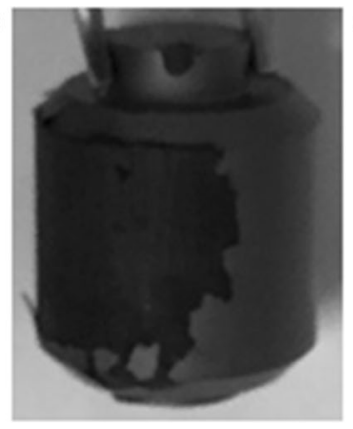

(b)

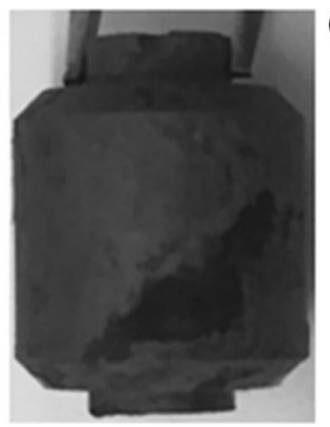

(c)

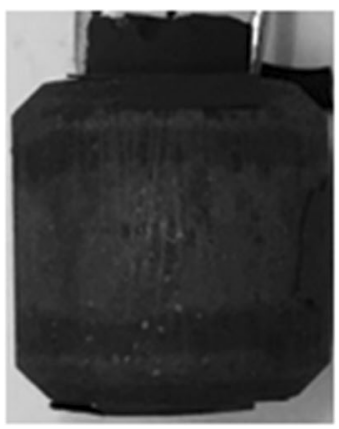

Fig. 9 CMSX-4 samples after a 25-h and b 100-h hot corrosion exposure with 25-h deposit recoat cycles. c 100-h hot corrosion exposure with 100-h deposit recoat cycles

\section{Limitations of Testing with CMSX-4}

CMSX-4 was developed for use in hotter stages of gas turbines, typically while coated. As such, this alloy contains significant amount of the refractory elements (see Table 1) which are needed to maintain mechanical strength. By contrast, the fraction of reactive elements (e.g., $\mathrm{Cr}, \mathrm{Al}, \mathrm{Si}$ ) required for the formation of stable, protective oxides are relatively low. Alloys with longer incubation periods may show different responses to variable deposit recoat cycle length.

In gas turbine applications, CMSX-4 is often used coated. This acts to protect the alloy from the potentially highly aggressive environments in which it is immersed. As such, the rapid metal loss rates recorded are unlikely to be representative of those found in engine operation.

\section{Conclusions}

Hot corrosion exposures were conducted on CMSX-4 to test the effect of changing the deposit recoat cycle length on final measured GML. This variable often changes between tests, but is rarely independently studied.

The results for CMSX-4 for all deposit recoat cycle lengths suggest very small corrosion differences when using different deposit recoat cycle lengths. Certainly these differences are insignificant compared to the spread in GML found around or between samples.

There are several practical impacts of this result. Firstly, the lack of observed variation of CMSX-4 hot corrosion damage with different deposit recoat cycle lengths indicates that CMSX-4 datasets obtained from experiments with different deposit recoat cycle lengths can be compared with confidence. Secondly, where tests are interrupted to insert additional samples, there is little notable impact for CMSX-4 under the tested conditions.

Further tests are underway for other material systems. There is also interest in longer test exposure times. 
Acknowledgements JS and SG would like to thank Rolls Royce Plc for sample supply.

Open Access This article is distributed under the terms of the Creative Commons Attribution 4.0 International License (http://creativecommons.org/licenses/by/4.0/), which permits unrestricted use, distribution, and reproduction in any medium, provided you give appropriate credit to the original author(s) and the source, provide a link to the Creative Commons license, and indicate if changes were made.

\section{References}

1. H. Zhou, P. A. Jensen and F. J. Frandsen, Fuel 86, 1519 (2007).

2. C. Duret-Thual, R. Morbioli and P. Steinmetz, in A Guide to the Control of High Temperature Corrosion and Protection of Gas Turbine Materials, ed. O. Morocutti (Commission of the European Communities, Belgium, 1986).

3. BSI Standards, Standard Number: BS ISO 17224:2015, BSI Standards Ltd, 2015.

4. BSI Standards, Standard Number: BS ISO 17248:2015, BSI Standards Ltd, 2015.

5. BSI Standards, Standard Number: BS ISO 21608:2012, BSI Standards Ltd, 2012.

6. J. Tomeczek, H. Palugniok and J. Ochman, Fuel 83, 213 (2004).

7. M. N. Richards and J. Stringer, British Corrosion Journal 8, 167 (1973).

8. N. J. Simms, P. J. Smith, A. Encinas-Oropesa, S. Ryder, J. R. Nicholls and J. E. Oakey, in Proceedings of ECF Workshop 2001, vol. 34, eds. M. Schütze, W. J. Quaddakers and J. R. Nicholls (Maney Publishing, London, 2001), p. 247.

9. J. R. Nicholls, N. J. Simms and A. Encinas-Oropesa, Materials at High Temperatures 24, 149 (2007).

10. F. Pettit, Oxidation of Metals 76, 1 (2011).

11. N. J. Simms, J. R. Nicholls and J. E. Oakey, Materials Science Forum 369, 833 (2001).

12. J. Sumner, A. Encinas-Oropesa, N. J. Simms and J. R. Nicholls, Materials and Corrosion 65, 188 (2014).

13. BSI Standards, Standard Number: BS ISO 26146:2012, BSI Standards Ltd, 2012.

14. J. Sumner, A. Encinas-Oropesa, N. J. Simms and J. R. Nicholls, Oxidation of Metals 80, 553 (2013). 